\title{
An Improved ID3 Decision Tree Algorithm Based on Attribute Weighted
}

\author{
Xian Liang ${ }^{1}$, Fuheng $\mathrm{Qu}^{1}$, Yong Yang ${ }^{1}$ \\ ${ }^{1}$ College of Computer Science and Technology, \\ Changchun University of Science and Technology, \\ Changchun 130022, China
}

\author{
Hua $\mathrm{Cai}^{2}$ \\ ${ }^{2}$ College of electronic and information engineering, \\ Changchun University of Science and Technology, \\ Changchun 130022, China
}

\begin{abstract}
ID3 decision tree algorithm uses information gain selection splitting attribute tend to choose the more property values, and the number of attribute values can not be used to measure the attribute importance, in view of the above problems, a new method is proposed for attribute weighting, the idea of simulation conditional probability, calculation of the close contact between the attributes and the decision attributes, as the attribute weights and combination with attribute information gain to selection splitting attribute, improve the accuracy of decision results. Experiments show that compared with the improved algorithm and the traditional ID3 algorithm, decision tree model has higher predictive accuracy, less number of leaves.
\end{abstract}

Keywords-decision tree; ID3 algorithm; information gain

\section{INTRODUCTION}

ID3 algorithm ${ }^{[1,2,3,4]}$ to select non leaf node in the decision tree model by introducing the definition of information gain in information theory, property has a better theoretical foundation but tend to choose the values of more, and the attribute importance from the number of attribute values cannot be measured. Aiming at the shortcomings of ID3 algorithm, The document[5]to introduce the concept of attribute importance to overcome the disadvantage of ID3 algorithm for attribute selection criteria. In document [6],the extended attributes selected with the proposed algorithm maximized the ranking mutual information between the candidate attributes and the decision attribute, and also minimized the ranking mutual information between the candidate attributes and the selected conditional arrtibutes on the same branch. The use of conditional probability calculation of close contact between the attributes and the decision attributes, as the weights and combined with information gain choose the split attribute, get the improved algorithm of ID3 decision tree algorithm based on attribute weighted.

\section{AN IMPROVED ID3 Decision TREe AlgorithM BASED ON ATTRIBUTE WEIGHTED}

Using information gain as a measure of the ID3 algorithm, without considering the relationship between classification and attribute, in the practical application have close ties between the two, the improved algorithm is proposed based on the shortcoming. The relevant definitions as: the sample data set $\mathrm{S}$, the classification properties $\mathrm{C}$ has $\mathrm{m}$ different values $\left\{C_{1}, C_{2}, \ldots, C_{m}\right\}$, the data set of $\mathrm{S}$ is divided into m subsets $S_{i} \quad(\mathrm{i}=1,2 \ldots \mathrm{m})$, description attribute $\mathrm{A}$ has $\mathrm{v}$ different values $\left\{A_{1}, A_{2}, \ldots, A_{v}\right\}$,description attribute $\mathrm{A}$ set $\mathrm{S}$ is partitioned into v subsets $S_{j} \quad(\mathrm{j}=1,2 \ldots \mathrm{v})$, the connection between the attribute $\mathrm{A}$ and classification attribute $\mathrm{C}$ is defined as $F_{A}=\sum_{j=1}^{v} \frac{\left|S_{j}\right|}{|S|} \cdot W_{j}$, The number of samples about the value of attribute $\mathrm{A}$ is $A_{j}$ is $\left|S_{j}\right|$, The total number of samples is $|S|$,the sum of probability of classification results is $W_{j}$, about the subset of samples under the condition of the value of attribute $\mathrm{A}$ is $A_{j}$. The way Calculation of the value of $W_{j}$ as: the number of the value of attribute $\mathrm{A}$ is $A_{j}$ is $\mathrm{t}$, there are $\mathrm{t}$ sample $\left\{m_{1}, m_{2}, \ldots, m_{t}\right\}$, any one sample $m_{j}(\mathrm{j}=1,2 \ldots \mathrm{t})$ class label attribute value for $C_{i}$, the obtained t samples under the condition of the value of attribute $\mathrm{A}$ is $A_{j}$, the probability of the value of class label attribute is $C_{i}$ in the t samples is $\frac{\left|C_{i j}\right|}{t} \cdot\left|C_{i j}\right|$ is the number of samples in the condition of the value of the categorical attribute is $C_{i}$ in the t samples. The same method can be calculated the probability in the condition of the value of classification attribute is $C_{i}$ in the data sets of the value of other description attribute in the samples $m_{j}$ get the corresponding sample subset, in the samples each description attribute values are independent of each other, the probability of according to the sample $m_{j}$ conjecture classification attribute values of $C_{i}$, the probability value $P_{j}$ is equal to the product of the probability of each description attribute in sample $m_{j}$ according to their own 
values get subset of data under the condition of the value of classification attribute is $C_{i}$. under the condition of the attribute $\mathrm{A}$ value for $\mathrm{B}$ corresponding the subset of data , the sum of the probability of correct introduced classification attribute's value in the subset of data is $W_{j}=\sum_{j=1}^{t} P_{j} \quad$.The use of conditional probability Calculation of close contact between the attributes and the decision attributes, as the weights and combined with information gain choose the split attribute, if a property to make the $M_{A}=\operatorname{Gain}(A) \cdot F_{A}$ value of the largest, then choose it for the splitting attribute. For example, there are a lot of value attribute id, it is the information gain is large, but it used to build classification model does not have any significance, using the method proposed in this paper to calculate the value of the relationships between Description attribute ID and classification attribute is small, so the value of $M_{\text {id }}=\operatorname{Gain}(\mathrm{id}) . F_{\text {id }}$ is small, avoid selecting properties such as the attribute id(the attribute value is more, but it is not important attributes ) as the decision node of decision tree model.

\section{THE EXPERIMENT RESUlTS ANALYSIS}

The data set in table 1, with establishing the decision tree use the ID3 algorithm and the improved ID3 algorithm.

TABle 1 The DATA SeT

\begin{tabular}{|c|c|c|c|c|c|}
\hline id & chinese & mathematics & english & physics & Summary \\
\hline 1 & general & good & bad & general & qualified \\
\hline 2 & general & good & good & good & qualified \\
\hline 3 & good & general & general & good & qualified \\
\hline 4 & optimal & general & good & good & qualified \\
\hline 5 & general & general & general & general & qualified \\
\hline 6 & good & bad & general & bad & unqualified \\
\hline 7 & optimal & bad & bad & general & unqualified \\
\hline 8 & good & optimal & optimal & optimal & qualified \\
\hline 9 & general & general & optimal & good & qualified \\
\hline 10 & optimal & bad & general & general & qualified \\
\hline 11 & bad & good & good & bad & unqualified \\
\hline 12 & good & general & good & good & qualified \\
\hline 13 & general & bad & good & general & qualified \\
\hline 14 & general & general & optimal & good & qualified \\
\hline 15 & good & bad & good & general & qualified \\
\hline 16 & optimal & general & optimal & good & qualified \\
\hline 17 & optimal & optimal & optimal & optimal & qualified \\
\hline 18 & good & bad & good & general & qualified \\
\hline 19 & good & general & bad & optimal & qualified \\
\hline 20 & general & general & general & general & qualified \\
\hline
\end{tabular}

ID3 algorithm build a decision tree as shown in figure 1.

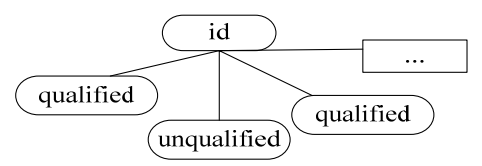

Figure 1. ID3 algorithm for constructing decision trees

Improved ID3 algorithm to construct the decision tree: Description attribute weights respectively:

$\mathrm{F}$ (chinese) $=3.66985$.

$\mathrm{F}$ (mathematics) $=3.9352$.

$\mathrm{F}$ (english) $=2.7904$.

$\mathrm{F}$ (physics) $=3.6665$.

$\mathrm{F}(\mathrm{id})=0.5643$.

The product of attribute weights and information gain of description attribute respectively:

$\mathrm{M}$ (chinese $)=0.222 * 3.66985=0.8147$.
$\mathrm{M}($ mathematics $)=0.197 * 3.9352=0.7752$.

$\mathrm{M}($ english $)=0.292 * 2.7904=0.8148$.

$\mathrm{M}($ physics $)=0.392 * 3.6665=1.4373$

$\mathrm{M}(\mathrm{id})=0.61 * 0.5643=0.3443$.

The improved ID3 algorithm choice of properties physical as the decision tree as shown in figure 2 .

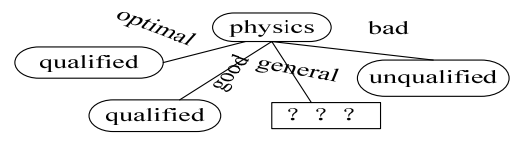

Figure 2. Improved ID3 algorithm for constructing decision subtrees 1

Improved ID3 algorithm build a decision tree as shown in figure 3. 


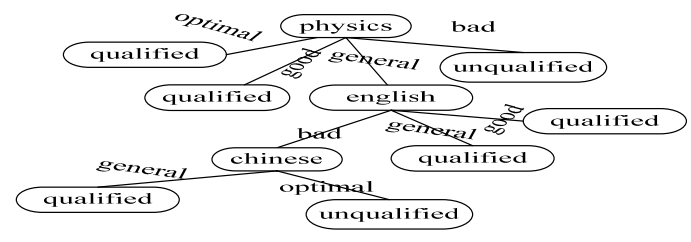

Figure 3. The decision tree built by improved ID3 algorithm

The compared to the decision tree of two kinds of algorithm, improved ID3 algorithm is not sensitive to more attribute values, build the decision tree has a lot of path, the categorization of data more detailed, to improve the decision tree classification accuracy.

In order to evaluate the performance of the improved ID3 algorithm, the experiment using seven data sets of UCI. Each data set $70 \%$ as the training data $30 \%$ as test data, the training data set is used to construct the decision tree model, the test data set is used to predict accuracy of the decision tree model. To test the traditional ID3 algorithm and the improved ID3 algorithm, the comparison results as shown in table 2 .

TABLE 2 EXPERIMENTAL RESULTS COMPARING

\begin{tabular}{|c|c|c|c|c|}
\hline \multirow[t]{2}{*}{ Data sets } & \multicolumn{2}{|c|}{ traditional ID3 algorithm } & \multicolumn{2}{|c|}{ improved ID3 algorithm } \\
\hline & Number of leaf nodes & correct $(\%)$ & Number of leaf nodes & correct $(\%)$ \\
\hline Breast-cancer & 93 & 91 & 84 & 91 \\
\hline Balance-scale & 241 & 24 & 221 & 31 \\
\hline Car Evaluation & 119 & 87 & 131 & 92 \\
\hline Nursery & 372 & 89 & 359 & 96 \\
\hline Adult & 386 & 74 & 375 & 82 \\
\hline
\end{tabular}

The table 3 shows that, improved ID3 algorithm compared with the traditional ID3 algorithm of decision tree model accuracy is higher, the number of leaf nodes is less. Experiments show that, improved ID3 algorithm solves the ID3 algorithm tend to choose more but not important attribute values, to improve the prediction accuracy of the decision tree classification model.

\section{CONCLUSIONS}

It is a project supported by the natural science foundation of Jilin Province(201215145) and the Twelfth Five-Science and Technology Research Projects of Education Department of Jilin Province (Grant No. 2013-420).The improved ID3 algorithm calculation of the close contact between the attributes and the decision attributes, as the attribute weights and combination with attribute information gain to selection splitting attribute, this method does not affect the weights of attribute is important and more attribute values is selected as split attribute, adds the weights of attributes of important but less attribute value is selected as split attribute, ensure that each time select important rather than more attribute values to construct the decision tree model, get higher prediction accuracy, less number of leaf nodes of the decision tree model.

\section{ACKNOWLEDGEMENTS}

It is a project supported by the natural science foundation of Jilin Province(20130101179JC-13) and the natural science foundation of Jilin Province(201215145) the Twelfth Five-Science and Technology Research Projects of Education Department of Jilin Province (Grant No. 2013-420). The corresponding author is Fuheng Qu.

\section{REFERENCES}

[1] Davidson Ian, Tayi Giri. Data preparation using data quality matrices for classify-cation mining. European Journal of Operational Research 2009,197(2): 764-772.

[2] Cheng Jiajun, Su Shoubao, Xu Lihua. Decision tree optimization algorithm based on multiscale rough set model, Journal of Computer Applications,2011,31(12):3243-3246.

[3] QUINLAND $\mathrm{J}$ R.Induction of decision trees[J],Machine Learning,1981,1(1): 81-106.

[4] Lu zhao,Cheng Shiping.Applicaion of machinery manufacturing decision-making based on ID3 algorithm[J],Journal of Computer Applications, 2011,31(11):3087-3090.

[5] Yu Jinping,Huang Ximei,Li Kangsun.Improved ID3 algorithm based on new attributes selection criterion[J],Application Research of Computers, 2012,29(8):2895-2899.

[6] Pan Pan,Wang Xizhao,Zhai Junhai.An improved induction algorithm based on ordinal decision tree[J],2014,44(1):41-44. 